\title{
Characterization of the bovine type I IFN locus: rearrangements, expansions, and novel subfamilies Angela M Walker ${ }^{1,3}$ and R Michael Roberts*2,3
}

\author{
Address: ${ }^{1}$ Department of Veterinary Pathobiology, University of Missouri, Columbia, MO 65211, USA, ${ }^{2}$ Department of Animal Sciences, \\ University of Missouri, Columbia MO 65211, USA and ${ }^{3}$ Christopher S Bond Life Sciences Center, University of Missouri, Columbia, MO 65211, \\ USA \\ Email: Angela M Walker - walkeram@missouri.edu; R Michael Roberts* - robertsrm@missouri.edu \\ * Corresponding author
}

Published: 24 April 2009

BMC Genomics 2009, 10:187 doi:10.1/86/147|-2164-10-187
Received: 17 September 2008

Accepted: 24 April 2009

This article is available from: http://www.biomedcentral.com/147/-2/64/10/187

(C) 2009 Walker and Roberts; licensee BioMed Central Ltd.

This is an Open Access article distributed under the terms of the Creative Commons Attribution License (http://creativecommons.org/licenses/by/2.0), which permits unrestricted use, distribution, and reproduction in any medium, provided the original work is properly cited.

\begin{abstract}
Background: The Type I interferons (IFN) have major roles in the innate immune response to viruses, a function that is believed to have led to expansion in the number and complexity of their genes, although these genes have remained confined to single chromosomal region in all mammals so far examined. IFNB and IFNE define the limits of the locus, with all other Type I IFN genes except IFNK distributed between these boundaries, strongly suggesting that the locus has broadened as IFN genes duplicated and then evolved into a series of distinct families.

Results: The Type I IFN locus in Bos taurus has undergone significant rearrangement and expansion compared to mouse and human, however, with the constituent genes separated into two sub-loci separated by $>700 \mathrm{~kb}$. The IFNW family is greatly expanded, comprising 24 potentially functional genes and at least 8 pseudogenes. The IFNB $(n=6)$, represented in human and mouse by one copy, are also present as multiple copies in Bos taurus. The IFNT, which encode a non-virally inducible, ruminant-specific IFN secreted by the pre-implantation conceptus, are represented by three genes and two pseudogenes. The latter have sequences intermediate between IFNT and IFNW. A new Type I IFN family (IFNX) of four members, one of which is a pseudogene, appears to have diverged from the IFNA lineage at least 83 million years ago, but is absent in all other sequenced genomes with the possible exception of the horse, a non-ruminant herbivore.

Conclusion: In summary, we have provided the first comprehensive annotation of the Type I IFN locus in Bos taurus, thereby providing an insight into the functional evolution of the Type I IFN in ruminants. The diversity and global spread of the ruminant species may have required an expansion of the Type I IFN locus and its constituent genes to provide broad anti-viral protection required for foraging and foregut fermentation.
\end{abstract}

\section{Background}

Viruses are constantly evolving to find more effective means to survive and multiply in their host species [1-3]. The immune defense system, in turn, exists in a perpetual state of co-evolution with the pathogens to limit infec- tious disease, a circumstance often likened to an "arms race." The primary defense mechanism against viruses in vertebrates is Type I IFN (interferon) of the innate immune system [4]. It can reasonably be argued that complex organisms like mammals can only survive as long as 
immune defenses can adjust to the strategies of invading pathogens. Accordingly, a rapidly evolving, adaptable IFN system is essential to mammals if they are to endure viral infections. Type I IFN are also pleiotropic cytokines, with significant roles in modulating adaptive immunity, cell proliferation and cell death, and numerous other processes vital to mammalian health and survival [1]. Most likely as a response to these challenges, Type I IFN demonstrate a complex evolutionary history that has resulted in the divergence of at least eight distinct subfamilies: IFNkappa (IFNK), IFN-beta (IFNB), IFN-epsilon (IFNE), IFNdelta (IFND), IFN-zeta (IFNZ), IFN-alpha (IFNA), IFNomega (IFNW), and IFN-tau (IFNT) [5].

Mammalian Type I IFN probably emerged during tetrapod evolution from an older cytokine family, Type III IFN, which provides the primary viral defense mechanism in fish $[6,7]$. It is difficult to determine exactly when Type I and Type III IFN diverged because no Type I IFN has been identified in amphibians, but the split definitely occurred prior to the divergence of birds and mammals approximately 310 million years ago (MYA) [5,8]. Type III IFN, known more commonly in mammals as either IFNlambda (IFNL) or interleukin (IL)28 and IL29, is encoded by a five exon gene, opposed to the single exon Type I IFN, and acts through a different receptor complex than Type I IFN $[9,10]$. Despite these differences, both Type I and Type III IFN have similar mechanisms of induction, activate the same signaling pathways, and trigger the same biological actions in the target cell [11]. Type III IFN has been retained in some mammalian species including humans and mice but has been lost in others [12]. Even when present, it appears to have assumed a less dominant role as an antiviral agent [11] and may have been supplanted as major player in antiviral defense with the emergence of contemporary Type I IFN.

All Type I IFN elicit an antiviral response, but some may play a more dominant role as first responders than others. IFNA and IFNB were the first Type I IFN to be characterized in human and have been assumed to constitute and the primary viral defense mechanism $[13,14]$. IFNA is released by almost all cell types and a few of its family members, specifically human IFNA2a and IFN2b, are currently approved for treatment of a range of viral diseases including hepatitis $\mathrm{B}$ and $\mathrm{C}$, condylomata acuminate (genital warts), and AIDS-related Kaposi sarcoma [15]. IFNB is the main IFN secreted by fibroblasts in response to a viral challenge, but is clearly produced by multiple cell types [16]. It acts in the immediate antiviral response and helps regulate the later expression of several IFNA [17]. IFNW and IFNZ both appear to have developed specific niches in antiviral protection for certain species. IFNW has been implicated in protection against specific viruses, such as parvovirus, particularly in cats $[18,19]$, while murine IFNZ provides a unique combination of high antiviral activity with relatively low lymphomyeolosuppresive activity [20], suggesting it may act to suppress viruses targeting the bone marrow and spleen. IFNK is predominately expressed in keratinocytes where it is acts through a unique cell-associated viral protection mechanism $[21,22]$. IFNE is expressed in a variety of cell types, but has been suggested on the basis of rather meager evidence to serve a specific role in reproductive tissues either in viral protection or early placental development $[5,23]$. IFND and IFNT, on the other hand, are not induced by viruses but instead are released by the early pre-implantation embryos of swine and ruminant species, respectively, where they appear to trigger responses in maternal uterine endometrium that allow the pregnancy to become established $[24,25]$.

The arrangement of Type I IFN genes within the locus likely reflects the origins and subsequent evolution of individual family members. All Type I IFN in human and mouse are clustered in an approximately $400 \mathrm{~kb}$ length of DNA, located on the short arm of chromosome 9 (9p21) in human and on the centromere-proximal region of chromosome 4 (4C4) in mouse [26-28]. Two genes of ancient origin, IFNB and IFNE, define the outer limits of the locus. All the other Type I IFN genes, except IFNK, are distributed between these two ancient genes, indicating the locus has expanded internally as IFN genes duplicated and then evolved into their respective families [27]. However, species-specific expansion and contraction of families has occurred, with some IFN families only existing in certain taxonomic groups. For example, IFND has only been identified in the pig and is absent in the mouse and human, while IFNZ is represented in the mouse, but only remnants of the gene has been found in rats, while it is completely absent in humans $[20,25,29]$. The IFNW, which are considered to have arisen from the IFNA at least 129 MYA $[16,30]$, constitute a particularly variable grouping. A single functional IFNW and at least two pseudogenes are present in humans, but only a single pseudogene can be identified in mice [27]. Even more bewildering, the family appears to have expanded in cats, which, on the basis of cDNA evidence, possess at least 10 variants [31], but not even a relic of the open reading frame can be found in the related carnivore, the dog [32]. Ruminant species, such as cattle, are known to possess several, apparently functional, IFNW $[33,34]$. There is also one example of a Type I family, the IFNT, that arose relatively recently (36 MYA) in the lineage to the ruminant artiodactyls. As a consequence, the IFNT are absent from all species except those in the sub-order Ruminantia $[33,35]$. Together, these data suggest that novel IFN genes can be gained and existing genes discarded in response to specific environmental challenges, which most likely include threats from emerging new pathogens. In addi- 
tion, existing IFN may become co-opted into new roles unrelated to viral pathogenesis, as has occurred in the case of the IFND $[20,25,29]$.

Although it has been clear for some time that there are similarities in the organization of the Type I IFN locus of cattle and that of other species [36,37], it was equally evident that the bovine locus must have some unique features, most notably because of the existence of the IFNT, genes unique to ruminant species whose protein products, although active in antiviral assays, have a primary role as hormones of pregnancy [38]. Cattle also have multiple IFNB while all non-ruminant species so far examined possess only a single copy IFNB [5]. Together these findings suggest either a decreased restriction on duplication of Type I IFN genes in cattle or evolutionary pressure to acquire additional genes. The recent sequencing of the bovine genome has provided the first opportunity for a detailed study of the Type I IFN locus in a ruminant species. Here we provide a detailed description and full annotation of the bovine locus and some inferences about its evolutionary history.

\section{Methods}

Annotation

Most of the IFN gene candidates were identified through the National Center for Biotechnology and Information (NCBI)'s bovine genome resource by using the basic local alignment search tools (BLAST) http:www.ncbi.nlm.nih.gov/genome/seq/BlastGen/Blast Gen.cgi?taxid=9913[39]. Additional searches were performed through NCBI by using the appropriate genome resource http://www.ncbi.nlm.nih.gov/mapview/[40] for other species, which are discussed later in this section, and by using the basic nucleotide BLAST suite http:// www.ncbi.nlm.nih.gov/blast/

Blast.cgi?CMD=Web\&PAGE TYPE=BlastHome $[41,42]$.

Several combinations of BLAST algorithms and databases within NCBI were utilized for this work and are described below [43].

\section{BLAST algorithms}

1. MegaBLAST was designed to compare highly related nucleotide sequences and works best when the target sequence has a $95 \%$ identity or higher to the query sequence.

2. Cross-species megaBLAST, also referred to as discontiguous BLAST, is a derivative of megBLAST that ignores certain bases, thereby allowing mismatches. It was designed to compare nucleotide sequences from one species to nucleotide sequences in another species.

3. BLASTN also compares nucleotide query sequences to a nucleotide database. This algorithm is slower than megaB-
LAST, but it can identify shorter sequence matches than megaBLAST. It was not specifically designed for cross-species comparisons.

4. TBLASTN was designed to compare a protein sequence with a nucleotide database dynamically translated in all reading frames.

\section{NCBI databases}

1. The "genome (reference)" database represents the most current publicly available assembly of a genome. The most current assembly of the bovine genome at the time this work was completed was assembly 3.1. The most current assembly for other species examined in this work are placed in parenthesis here - human (36.2), mouse (37.1), horse (1.1), and dog (2.1).

2. The "WGS contigs" database contains the contigs, or overlapping unassembled sequences, that forms the basis for the assembled genome. Both pig and cat do not have an assembled genome available at this time and only the "WGS contigs" database could be searched for genomic information for these species.

3. The "traces-WGS" database contains the trace data for whole genome shotgun sequence (WGS) bacterial artificial chromosome (BAC) end sequencing. This database contains single pass sequencing reads that are not trimmed based on quality or vector contamination.

4. The "nucleotide collection (nr/nt)" database contains all Genbank, RefSeq, EMBL (Europe's primary nucleotide database), DNA Database of Japan (DDJB), and many Protein Databank (PDB) sequences. The "nucleotide (nr/ $\mathrm{nt})$ " database is subdivided into "human nucleotide (nr/ nt)," "mouse nucleotide (nr/nt)," and "others nucleotide (nr/nt)" databases. The "others nucleotide (nr/nt)" database does not contain any mouse or human sequences.

Bovine IFNB, IFNA, IFNW, and IFNT CDNA sequences (Table 1) from GenBank were used to perform a megaBLAST search in the bovine "genome (resource)" database. Human IFNE, murine IFNZ, porcine IFND, human IFNK, and human IFNL/IL28-29 sequences (Table 1) were queried with cross-species megaBLAST in the bovine "genome (reference)" database because no bovine homologues for the latter group of genes have been reported. The translated sequence for each of the non-bovine IFN CDNA were also queried with TBLASTN in the bovine "genome (reference)" database because the TBLASTN algorithm can often identify homologues that are not detected through other searches. IFNL/IL28-29 nucleotide and amino acid sequence were also queried in the bovine "WGS contig" database by using a cross-species megaBLAST and TBLASTN search, respectively, to verify no sequence with 
Table I: Query sequences used for the genomic searches.

\begin{tabular}{|c|c|c|c|c|c|}
\hline Species & Gene & Accession No. & Species & Gene & Accession No. \\
\hline Bovine & IFNA & AY325272 & Bovine & IFNT & AFI96324 \\
\hline Bovine & IFNA & M10954 & Porcine & IFND & $\underline{Z 22707}$ \\
\hline Bovine & IFNA & AY523531 & Porcine & IFND & $\overline{Z 22706}$ \\
\hline Bovine & IFNA & DQ396807 & Human & IFNK & NM 020124 \\
\hline Bovine & IFNA & Z46508 & Human & IFNE & $\overline{N M \quad 176891}$ \\
\hline Bovine & IFNB & MI5478 & Human & IFNL & AY 184374 \\
\hline Bovine & IFNW & MII002 & Human & IFNL & AY 184373 \\
\hline Bovine & IFNT & M31557 & Human & IFNL & AY184372 \\
\hline Bovine & IFNT & $\mathrm{AF} 196320$ & Murine & IFNZ & NM 197889 \\
\hline Bovine & IFNT & $\overline{A F I 96322}$ & & & \\
\hline
\end{tabular}

high identity to IFNL/IL28-29 in the bovine genome was missed. Specific genes were analyzed in the bovine "traces-WGS" database to verify frameshift mutations or nucleotide variations from the query sequences.

The Bovine Genome Sequencing and Annotation Consortium created a consensus predicted gene set through an algorithm, termed GLEAN, developed during the annotation of the honey bee that used latent class analysis to automatically combine disparate gene prediction evidence [44]. Since the majority of positive megaBLAST, cross-species megaBLAST, and TBLASTN matches were clustered on two scaffolds, Chr8.25 [Genbank: NW 001495421] and Chr8.34 [Genbank:NW_001495430], all GLEAN models on those two scaffolds were also annotated through Apollo http:// apollo.berkeleybop.org/current/index.html $[45,46]$. In brief, Apollo is a genome annotation viewer and editor that was originally designed for the annotation of the Drosophila melanogaster genome. The Bovine Genome Sequencing and Annotation Consortium created input files for Apollo containing EST matches, cDNA matches, translated protein matches, and gene model data including all GLEAN models for the bovine genome assembly 3.1. GLEAN models present on scaffolds Chr8.25 and Chr8.34 that had not been identified in the aforementioned searches were queried through BLASTN and discontiguous megaBLAST in the "others nucleotide collection (nr/nt)" and "human nucleotide collection (nr/ nt)" databases to verify their status as IFN genes or another gene family. Discontiguous megaBLAST and TBLASTN searches in human, mouse, equine, porcine, feline, and canine "genomic (reference)" and "WGS contigs" databases were performed for the unique IFN family discovered during the annotation of Chr8.34.

The 64 identified IFN genes and pseudogenes and the original query cDNA from Genbank (Table 1) were aligned through CLUSTALW in BioEdit version 7.09 http:/ /www.mbio.ncsu.edu/BioEdit/BioEdit.html[47,48]. A pairwise comparison to known IFN nucleotide sequences was performed through the Maximum Composite Likelihood method in MEGA version 4 (MEGA4) http:// www.megasoftware.net/[49] to determine the IFN family for each gene [50,51].

IFNT was queried with megaBLAST in the bovine "tracesWGS" database to validate the number of IFN genes present in the genome. Bovine sequence matches that had greater than $94 \%$ sequence identities to the query IFNT for more than 400 basepairs (bp) were visually inspected. An IFNT match was counted as a positive if the sequence had greater than $98 \%$ identity to an IFNT cDNA in the portion of the trace with a quality score, available through NCBI, higher than 40 on a scale between 0 and 100. The total number of IFNT matches in the WGS contig database was divided by the bovine genome coverage to approximate the total IFNT gene number.

\section{Phylogenetic Reconstruction}

Alignments for the genomic IFN ORFs were created through ClustalW in BioEdit version 7.09, with individual genes denoted by their GLEAN numbers. Phylogenetic trees were constructed in MEGA4 through the Neighborjoining (NJ) method with bootstrapping test (1000 replicates). The tree was rooted to IFNK [5] and a second tree was created with the assumption of a non-uniform rate of change between sites (gamma $=1$ ).

\section{Identification of repetitive elements}

The localization and identity of all repetitive elements were determined by using the RepeatMasker program http://www.repeatmasker.org/[52], which uses the RepBase library of repeat elements [53]. Sub-locus 1, corresponding to 20000-711500 bp in scaffold Chr8.25, and sub-locus 2, corresponding to $2000-446000$ bp in scaffold Chr8.34, sequences were first selected through Apollo and imported into a word processing program, Microsoft Word. All gaps within the scaffolds, which are represented by an " $\mathrm{N}$ " in the bovine assembly, were removed manually. IFN sub-loci sequences were then analyzed in RepeatMasker version 3.1.9 run in default mode 
with blastp version 2.0MP-WashU http://blast.wustl.edu/ [54] to determine the percentage of repetitive elements. Bos taurus was set as the assumed species within the program parameters. Simple repeats and low complexity regions were not masked, which means they were not excluded as start sites for a BLAST match, and the matrix was optimized for $42 \%$ GC content based on sub-loci optimization pre-runs.

\section{Results and discussion \\ IFN Gene Families in Bos taurus}

Evidence for the presence of all previously known Type I IFN subfamilies except IFNZ was found on chromosome 8 of the bovine genome assembly 3.1 (Table 2). IFNZ has only been reported in mouse $[27,55]$, and so its absence in Bos taurus was anticipated. Both IFNK and IFNE are present as single genes with intact ORF and are assumed to be functional, providing the first evidence that either of these subfamilies is present in ruminants. Bovine IFNK and IFNE have $81.2 \%$ and $84.7 \%$ nucleotide identity respectively when compared to their human orthologues, values that are similar to their degree of conservation between human, cat, dog, and pig (A. Walker, unpublished data). The IFNW family is greatly expanded compared to other species that have so far been examined. There are 24 potential IFNW and at least 8 pseudogenes. The query IFNW [Genbank:M11002] [34] exactly matches two IFNW annotated from the genome database (GLEAN 09983 [Bovine Genome Database temporary ID:2733] and GLEAN 10004 [Bovine Genome Database temporary ID: 2525]). The remaining IFNW range in sequence identity from $86 \%$ to $96 \%$ relative to the query sequence.

Table 2: Cross-species comparison of IFN subfamilies.

\begin{tabular}{cccc}
\hline & \multicolumn{3}{c}{ Gene Number } \\
\cline { 2 - 4 } Subfamily & Human & Mouse & Cow \\
\hline IFNK & 1 & 1 & 1 \\
IFNE & 1 & 1 & 1 \\
IFNB & 1 & 1 & 6 \\
IFND & 0 & 0 & 0 \\
IFNZ & 0 & 2 & 0 \\
IFNA & 13 & 14 & 13 \\
IFNW & 1 & 0 & 24 \\
IFNT & 0 & 0 & 3 \\
IFNX & 0 & 0 & 3 \\
IFNL & 3 & 3 & 0 \\
\hline
\end{tabular}

The number of predicted IFN genes in each subfamily based on genomic analysis of the mouse, human, and bovine are shown. Predicted pseudogenes based on frameshift mutations or stop codons within the first 100 aa of the coding sequence have been excluded from the table. The bovine Type I IFN locus has an expansion of both the IFNB and IFNW subfamilies. Cattle have also acquired two novel IFN subfamilies, IFNT and a previously unidentified IFN family, termed IFNX, discovered during this analysis.
The IFNA and IFNB are also present in multiple copies, with 13 and 6 genes, respectively, although neither family is as large as the IFNW. An apparent IFNB pseudogene, deemed nonfunctional due to a frameshift deletion, also exists.

IFND are only represented as three pseudogenes, a not unexpected finding, as a functional gene has only been reported previously for the pig [56].

Three apparently functional IFNT are found within the locus. Surprisingly none of these provide an exact match for any of the many cDNA and gene sequences that have previously been reported. Previous mRNA sequencing of the IFNT family had indicated that at least 18 bovine IFNT might exist [57]. Only three IFNT are present in the bovine genome assembly 3.1, however. One particular, well established sub-family, the IFNT2 grouping [57], is not represented at all in the assembly. Additional analysis revealed 45 acceptable matches to IFNT in the WGS contig database. Since the bovine genome at this time has $7.1 \mathrm{X}$ coverage, the number of IFNT matches divided by this coverage value suggests the possibility of around six IFNT. One explanation is that these "extra" genes have been lost in the assembly process, but even this higher value is still significantly lower than the 10 to 18 IFNT previously believed to exist. Some of the latter are most likely alleles.

Most interestingly, we detected a novel Type I IFN, which, as we shall discuss later, consists of three potentially functional genes and one pseudogene, none of which provides a close sequence match with any previously described Type I IFN. For convenience, and until an appropriate nomenclature is approved, this new family will be termed IFNX.

A weak sequence identity to IFNL was found on chromosome 13, specifically located on scaffold Chr13.80 [Genbank:NW 001493172] from 635,850 to 636,120 bp. This sequence appears not to encode a functional gene in either the 3.1 assembly or the WGS contig database. These data suggest that the Type III IFN family exists only as a relic and is no longer a functional component in bovine pathogen defenses.

\section{Locus Map}

The Type I IFN locus is organized similarly in mouse and human, and possibly also in pig, i.e. there is relatively conserved synteny across rodents, primates, and swine. Two mammalian IFN genes of ancient origin, IFNB and IFNE, define the outer limits of the locus, with all the other genes, except IFNK, distributed between these two markers. The genes are predominantly (but not exclusively) localized on one strand and transcribed in the same direction as the IFNB and IFNE [27]. The relative 
arrangements of the murine and human versus the bovine IFN locus are illustrated in Fig. 1. The bovine Type I IFN locus is clearly organized differently than that of the other two species. Instead of a single stretch of DNA defining the locus, cattle have two sub-loci ( 1 \& 2) encompassing $701 \mathrm{~kb}$ and $441 \mathrm{~kb}$, respectively, separated by a gap estimated to be approximately 11 megabases (Mb) in assembly 3.1 (but $750 \mathrm{~kb}$ in assembly 4.0 ). The gene density based on the both predicted open reading frames (Gene Sequence map) and bovine EST and mRNA alignments with the assembled sequence (BT UniG map) is lower in the region between the two IFN sub-loci than much of the rest of chromosome 8 , but many genes are present and actively transcribed.

The following explanation of the locus organization has been based on the chromosome map assigned during the assembly process and reported by NCBI (Fig. 2). An IFNB defines the distal end (relative to the start of the chromosome map) of sub-locus 2 (Fig. 1 \&2). IFNE, while present in the bovine, is located towards the distal end of sublocus 1 . The majority of the genes in both sub-loci are transcribed in the same direction as the distally placed IFNB, except one cluster of IFNW and IFNA and the solitary IFNE, which are transcribed in the opposite direction. IFNK is present in a single "copy" nearer to the start of the chromosome map and well separated (6.044 Mb) from the closest sub-locus (sub-locus 1) (Fig. 1). The bovine IFNK location is very similar to that in the human where a single IFNK is located $6.5 \mathrm{Mb}$ from the Type I IFN locus [22] (Fig. 1).
There are three clusters of IFNA/IFNW. Two of them are on sub-locus 1 , one at the proximal end, the second placed about half way along (Fig. 2). A gene set in the first IFNA/IFNW cluster 1 is a palindrome to one in the second cluster. The corresponding gene pairs have complete nucleotide identity within their coding regions, suggesting that the duplication or gene conversion event that led to their formation occurred quite recently. The third cluster of IFNA/IFNW is at the distal end of sub-locus 2, but lacks the duplicated group of four genes in IFNA/IFNW clusters 1 and 2.

Only one non-IFN gene is detectable within sub-loci 1 and 2, an intronless kelch-like 9 (KLHL9) located $33.5 \mathrm{~kb}$ proximal to IFNA/IFNW cluster 2 in sub-locus 1 (Fig. 2). The orthologous KLHL9 gene can be found in the Type I IFN locus of the mouse approximately $25 \mathrm{~kb}$ from the nearest functional IFN (IFNA8) [27] and $29 \mathrm{~kb}$ from the nearest IFN (IFNA6) in human. The fact KLHL9 has resisted duplication despite residing close to genes undergoing multiple duplications is noteworthy and possibly indicates that multiple copies of this gene are not well tolerated.

The presence of KLHL9, which appears to be under different evolutionary constraints than IFNW and IFNA, close to IFNA/IFNW cluster 2 suggested the cluster as whole might be under different evolutionary control than cluster 1 or 3. Evolutionary divergence rates do not indicate that this is the case, however (Table 3 ). While cluster 1 does have a slightly higher divergence rate than cluster 2 , all

\section{Conserved Locus Schematic}
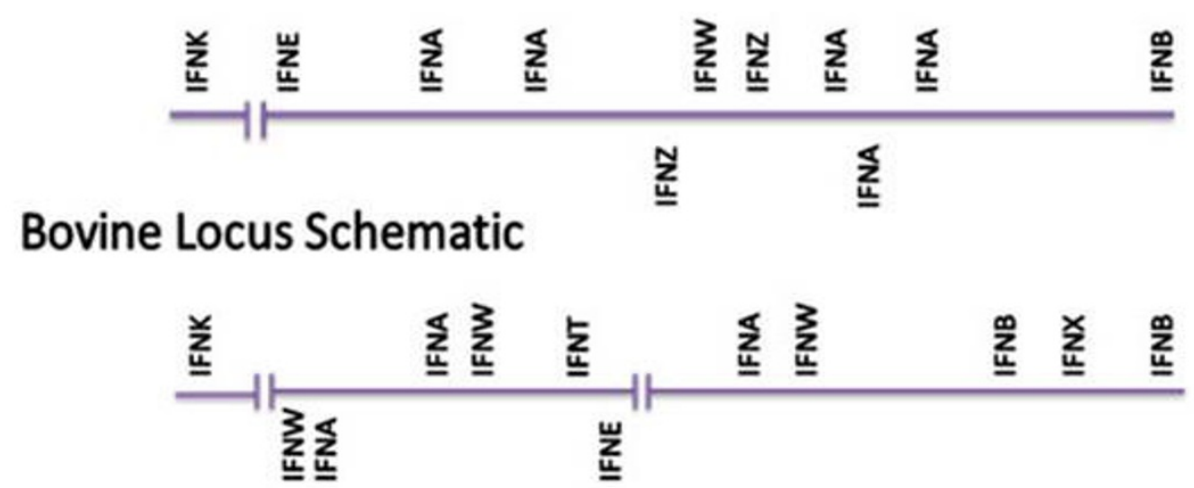

\section{Figure I}

Type I IFN locus schematics. These schematics, while not drawn to scale, illustrate the basic characteristics of the locus in mouse and human, the Conserved Locus Schematic, and the bovine locus. Each IFN gene is represented by its abbreviated name and its position above and below the schematic line represents the direction of its transcription. 


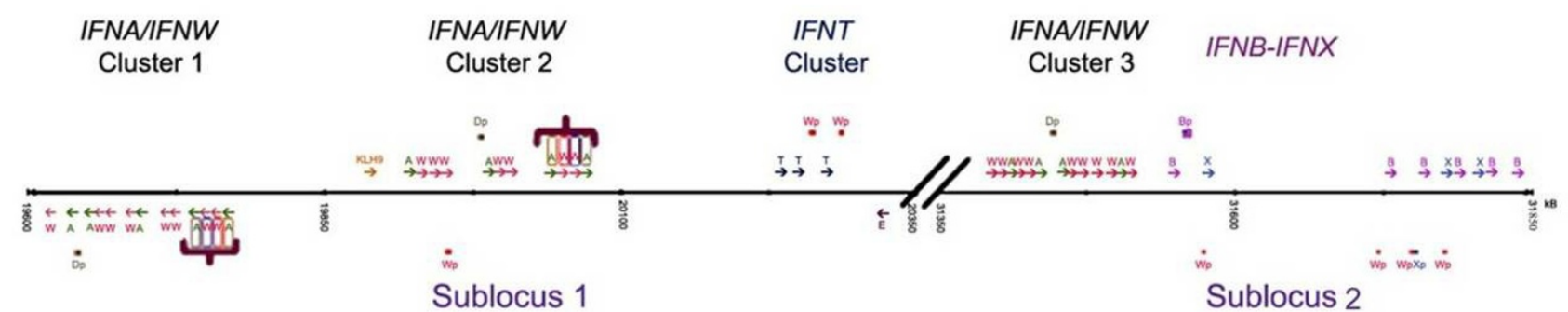

\begin{abstract}
Figure 2
Genomic map of the bovine Type I IFN locus. Blast searches of the bovine genome database revealed that all matches to known IFN genes, except IFNK, reside within two sub-loci, illustrated in the gene map shown in Fig. 2. Both the position of each gene relative to the line and the direction of the arrow on the map denote the direction of transcription. The subfamily for each gene is designated by the final letter of the abbreviated name. Pseudogenes are indicated by a box instead of an arrow and the letter "p" after the subfamily designation. Specific gene clusters have been labeled according to the subfamily or subfamilies they contain. A recent example of gene duplication is illustrated in the IFNWIIFNA cluster I and 2. The bracketed gene set in IFNA/IFNW cluster I is a palindrome to the bracketed gene set in the IFNA/IFNW cluster 2 with identical coding sequences for genes specified by correspondingly colored boxes.
\end{abstract}

three clusters are relatively constant in their rate of change. Inter-cluster divergence is actually very low in all three of the IFNA/IFNW clusters.

The IFNT and two IFNW pseudogenes are neighbors at the distal end of sub-locus 1 , suggesting that this cluster of genes originated from an IFNW that had become isolated from other IFNW before the divergence of the IFNT. Its unique position outside the IFNA/IFNW clusters and close to the edge of the sub-locus may have permitted the rapid expansion and evolution of the IFNT family without the restraints placed on the clustered IFNW.

All non-ruminant species examined to date, including mouse, human, cats, dogs, rabbits, and pigs, contain only

Table 3: Divergence within IFNA/IFNW clusters.

\begin{tabular}{cccc}
\hline & \multicolumn{3}{c}{ IFNA/IFNW Clusters } \\
\cline { 2 - 4 } Subfamily & I & 2 & 3 \\
\hline IFNW & $0.086 \pm 0.007$ & $0.071 \pm 0.007$ & $0.063 \pm 0.006$ \\
IFNA & $0.053 \pm 0.006$ & $0.04 \pm 0.006$ & $0.045 \pm 0.006$
\end{tabular}

Divergence rate \pm standard error for IFNA and IFNW within clusters was calculated by using the Maximum Composite Likelihood method. The number of base substitutions per site from averaging over all sequence pairs within each cluster is shown. Cluster I genes have a slightly higher divergence rate than cluster I and 2, but genes within all three clusters are relatively equal in their rate of change. one IFNB [16]. In cattle, this family has clearly expanded and extends from the distal end of IFNA/IFNW cluster 3 to the end of sub-locus 2 . Interspersed within these multiple IFNB are members of the previously unidentified IFN family, IFNX. Again, it is tempting to hypothesize that the IFNX and expanded IFNB family were able to emerge due to their location on the edge of the sub-locus 2, as suggested for the IFNT in sub-locus 1.

\section{Repetitive Elements within Sub-loci}

Repetitive elements have been implicated in gene duplication by creating regions predisposed to homologous recombination $[58,59]$ and also in organizing the assembly of enhanceasomes, as recently described for transcription of IFNB [60]. Over one third of the bovine Type I IFN locus consists of interspersed repeats, rather less than the $43 \%$ assessed for the murine Type I IFN locus [27]. Repeats are more enriched in sub-locus 1 largely because of the presence of a greater number of long interspersed nucleotide elements (LINE) and long terminal repeats (LTR) (Table 4). LINE2 elements are absent in both subloci. Short interspersed nucleotide elements (SINE)s are present in similar proportions, approximately $15 \%$, for both sub-loci. The arrangement of these elements provides no obvious insight into whether the sequences are involved in gene duplication or in controlling transcription. 


\section{Palindromic IFN within IFNA/IFNW clusters I and 2}

As mentioned previously, a gene set located on the distal end of IFNA/IFNW cluster 1 is a palindrome to a gene set on the distal end of cluster 2 (Fig. 3A). Two IFNA and two $I F N W$ are present in each gene set and these are designated as $A(1)$ and $A(2)$ for the two IFNA and $W(1)$ and $W(2)$ for the two IFNW in cluster $1 . A(1)$ and $A(2)$ are located at the proximal and distal ends of the gene set in cluster 1 , respectively. $W(1)$ and $W(2)$ are located from proximal to distal between $\mathrm{A}(1)$ and $\mathrm{A}(2)$. The genes with identical ORFs in cluster 2 are designated as $A(1) /, A(2) /$, $W(1)^{\prime}$, and $W(2)^{\prime}$ (Fig. 3B). The ORF and the first $350 \mathrm{bp}$ of the 3/ UTR for the two IFNA and IFNW gene pairs are identical, and an approximately 550 bp promoter for both IFNA gene pairs and the $W(2) / W(2) /$ pair are also identical. The promoters of the $W(1) / W(1) /$ pair, while closely similar (99\%), are not identical, however. All nucleotide differences between the $W(1) / W(1) /$ gene pair are within a region between 300 bp and 400 bp upstream of the transcriptional start site.

Table 4: Repetitive elements within the bovine Type I IFN subloci.

\begin{tabular}{lcc}
\hline & Sub-locus I & Sub-locus 2 \\
\hline SINEs: & $\frac{15.29 \%}{0.00 \%}$ & $\frac{15.68 \%}{0.00 \%}$ \\
\hline Alu/BI & $0.51 \%$ & $0.81 \%$ \\
MIRs & $17.74 \%$ & $\underline{11.23 \%}$ \\
LINEs: & $10.33 \%$ & $2.20 \%$ \\
LINEI & $0.44 \%$ & $0.29 \%$ \\
LINE2 & $0.04 \%$ & $0.00 \%$ \\
L3/CRI & $6.93 \%$ & $8.73 \%$ \\
RTE & & \\
LTR elements: & $\underline{4.01 \%}$ & $\underline{2.75 \%}$ \\
MaLRs & $0.89 \%$ & $0.98 \%$ \\
ERVL & $0.12 \%$ & $0.03 \%$ \\
ERV classI & $0.79 \%$ & $0.22 \%$ \\
ERV classII & $0.00 \%$ & $0.00 \%$ \\
DNA elements: & $\underline{2.36 \%}$ & $\underline{2.68 \%}$ \\
$\quad$ MERI_type & $1.72 \%$ & $2.45 \%$ \\
MER2_type & $0.45 \%$ & $0.00 \%$ \\
Unclassified: & & \\
Total interspersed repeats: & $0.00 \%$ & $0.00 \%$ \\
Small RNA: & $39.40 \%$ & $32.35 \%$ \\
\hline
\end{tabular}

The percentage of repetitive elements for the Type I IFN sub-loci was calculated in RepeatMasker 3.1.9. The length of nucleotide sequence encompassing the repetitive element divided by the length of the total nucleotide sequence for either sub-locus I or sub-locus 2 was used to calculate the percentage shown in each column. Sub-locus I has a higher proportion of LINEI and LTR elements than sub-locus 2 resulting in a higher total number of interspersed repeats within sublocus.
Two different evolutionary processes, either gene duplication or gene conversion, could possibly explain the existence of the IFNA/IFNW palindromic gene sets. Gene duplication involves the formation of a new gene copy. Gene conversion, on the other hand, does not generate new gene copies, but instead homogenizes existing genes. Both gene duplication and gene conversion have been specifically implicated in the evolution of the IFNA in human, chimpanzee, dog, rhesus monkey, rat, and mouse $[30,61,62]$. Gene conversion, specifically, was predicted by two different statistical programs, GARD and GENECONV, in humans, chimpanzee, rhesus monkey, and mice. Furthermore, despite IFNA genes aligning in conserved positions on a locus map for chimpanzees and humans, the subfamily separated into species-specific clades on phylogenetic analysis [61], strongly indicating gene conversion has occurred in the IFNA subfamily in these two species. Although gene duplications cannot be unambiguously distinguished from gene conversions [63], the latter seldom involve sequence longer than $1 \mathrm{~kb}$ in mammals, with $3 \mathrm{~kb}$ considered the maximum length [63]. Therefore, when the sequence tract involved is "too large" for gene conversion, gene duplication is usually implicated [64]. The palindomic gene set involves at least a $27 \mathrm{~kb}$ tract, far exceeding this size limit and reducing the likelihood of a conversion event. Therefore, a segmental duplication event, which is a specific type of gene duplication that involves a large segment of a locus, combined with an inversion is the best explanation for the palindrome [65].

\section{Selective Pressure on the ORF of Type I IFN Subfamilies}

Comparison of the rate of non-synonymous nucleotide change relative to the rate of synonymous change can provide information about the type of selection operating on the members of a multigene families [66]. If neutral selection is occurring, then all nucleotides in a sequence are equally likely to change. Consequently the rate of synonymous nucleotide changes (dS) will be equal to the rate of non-synonymous changes $(\mathrm{dN})$ and $\mathrm{dS}: \mathrm{dN}$ will equal 1 . Rapid change in the amino acid sequence is the desired endpoint for positive selective pressure. Hence, in this scenario, $\mathrm{dN}$ will exceed $\mathrm{dS}$, and $\mathrm{dN}$ : $\mathrm{dS}$ will be greater than 1 . Conversely, if strong selection against amino acid change is present (purifying selection), $\mathrm{dN}$ will be less than $\mathrm{dS}$ and $\mathrm{dN}$ : $\mathrm{dS}$ will be less than 1 . Virtually all pairwise comparisons within IFNA, whatever the species $[61,67]$, and IFNT [57] have shown the overall value for $\mathrm{dN}$ not to be significantly higher than $\mathrm{dS}$. Indeed, $\mathrm{dN}$ values have been generally calculated to be lower than $\mathrm{dS}$, consistent with the conclusion that there has not been strong positive selection for amino acid change within the coding regions of these subfamilies of IFN. 
A.

IFNA/IFNW Cluster 1

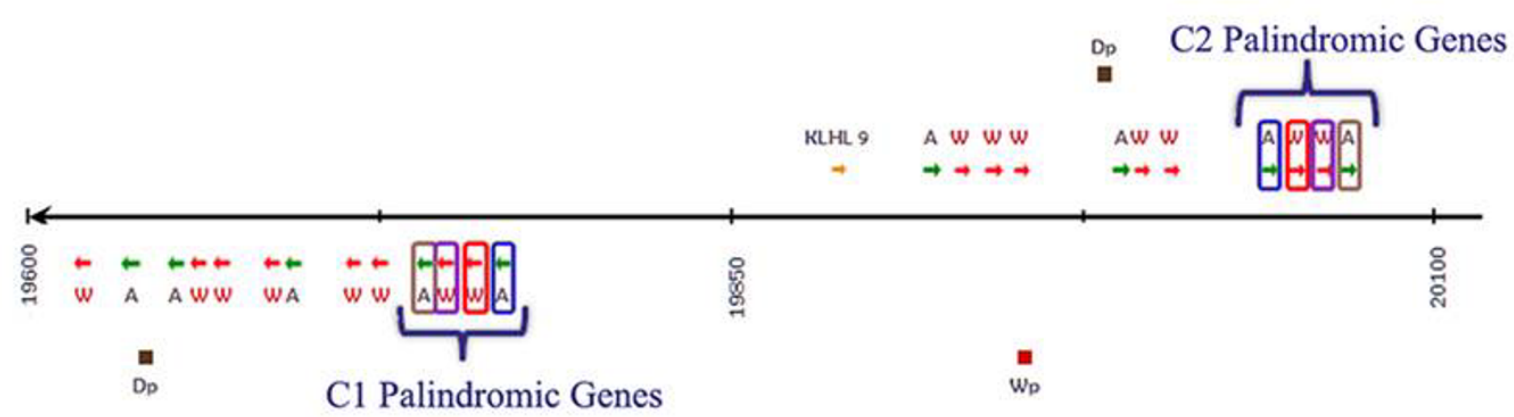

B.

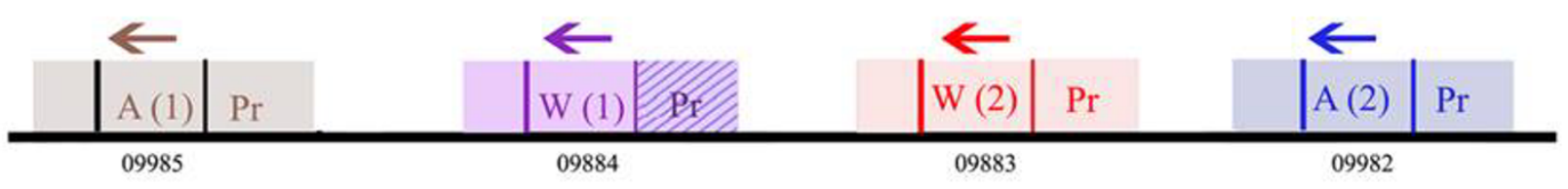

C1 Palindromic Genes

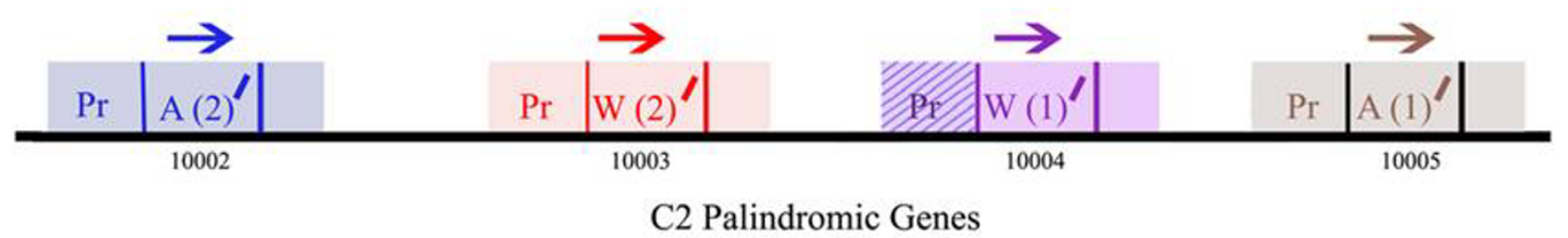

Figure 3

Palindromic gene sets within IFNA/IFNW clusters I and 2. (A) An enlarged image of IFNA/IFNW clusters I and 2 from Fig. 2 is shown. (B) The promoter (Pr), ORF, and 3/UTR for each gene have been depicted in a schematic of both gene sets (not drawn to scale). The direction of gene transcription is indicated by an arrow above each gene and the GLEAN number is written below each gene (GLEAN 0998I [Bovine Genome Database temporary ID:237I], GLEAN 09982 [Bovine Genome Database temporary ID:2258], GLEAN 09983 [Bovine Genome Database temporary ID:2733], GLEAN 09984 [Bovine Genome Database temporary ID:I340], GLEAN 10002 [Bovine Genome Database temporary ID:I I55], GLEAN I0003 [Bovine Genome Database temporary ID:22I7], GLEAN 10004 [Bovine Genome Database temporary ID:2525], GLEAN 10005 [Bovine Genome Database temporary ID:74]. Genes with 100\% nucleotide identity within their promoters, ORFs, and 3/UTRs are shown in matching solid colors. The only gene pair that does not have $100 \%$ nucleotide identity in the promoter, $\mathrm{W}(\mathrm{I}) / \mathrm{W}(\mathrm{I})^{\prime}$, is indicated by diagonal stripes through the promoter box.

The $\mathrm{dN}$ : $\mathrm{dS}$ for all multigene bovine IFN subfamilies, including only IFN annotated during this work, is illustrated in Fig. 4. The $\mathrm{dN}$ and $\mathrm{dS}$ values for every gene pair in a Type I IFN subfamily were calculated and plotted against each other. No multigene IFN subfamily (IFNA, IFNB, IFNW, or IFNT) in bovine has a dN significantly exceeding $\mathrm{dS}$. In fact, bovine IFNA and IFNW provide strong evidence for purifying selection. The significance of purifying selection within IFNW and IFNA subfamilies was also verified through a codon based Z-test ( $\mathrm{p}<$ 0.001 ), which determines selective pressure for a gene pair or group of genes based on the difference between $\mathrm{dN}$ and $\mathrm{dS}$ through a one-tailed t-test (Table 5). Four IFNB pairs out of 15 pairwise comparisons examined provide some 
evidence for purifying selection on the basis of the pairwise codon based Z-test (Table 6), but such selective pressures are not evident for the family as a whole $(\mathrm{p}=0.171)$. On the other hand, the same test employed for neutral selection provided no evidence for a lack of selective pressure operating on any members of the IFNB subfamily ( $\mathrm{p}$ $=0.343$ ), indicating the six genes comprising the bovine IFNB subfamily may be too few for a meaningful analysis. Since only three IFNT were identified, statistical analysis of this subfamily from the genomic data was not possible.

The classic model of gene duplication states that after a duplication event one gene continues to perform the ancestral function while the second either rapidly evolves to fill a new niche or becomes inactive $[65,68,69]$. As a consequence gene duplication is usually followed by a period in which there is an acquisition of non-synonymous nucleotide changes in one of the two genes, leading to a divergence in amino acid sequence. This temporary relaxation of purifying selection, in which $\mathrm{dN}$ : dS approaches 1, permits the gene to become fine-tuned to its new role or, more commonly, results in pseudogenization. Such a sequence of events does not appear to have occurred during the large scale expansion of the IFNW family where there is strong evidence for purifying selection operating on the coding regions of the genes (Fig. 4 $\&$ Table 5). One potential explanation is that sub-functionalization is occurring through alterations in the manner in which these genes are subjected to transcriptional control rather than in the structure of the proteins themselves. Evidence for positive selective pressure in the regulatory regions of IFNW would strongly support this hypothesis, but a detailed promoter analysis is beyond the scope of this work.

\section{IFNX: Evidence for a novel subfamily}

Based on the current assembly, the IFNX subfamily consists of three potential genes and one probable pseudogene. The origin of these genes is currently unclear, but they appear to constitute a unique IFN subfamily, whose closest relatives are the IFNA based on a Maximum Composite Likelihood divergence analysis, with IFNX sharing over

Table 5: Purifying selection within IFNW and IFNA coding regions.

\begin{tabular}{ccc}
\hline Family & p-Value & dS-dN \\
\hline IFNA & 0.0 & 3.824 \\
IFNW & 0.0 & 4.013 \\
\hline
\end{tabular}

The significance of purifying selection in the coding regions of IFNW and IFNA subfamilies was verified through a codon based Z-test analyzing the overall average for the subfamilies. The $\mathrm{p}$-value is shown in the second column with p-values less than 0.05 considered significant. The test statistic, the difference between $\mathrm{dN}$ and $\mathrm{dS}$, is shown in the third column. The variance of the difference was computed by using the bootstrap method (1000 replicates).
$65 \%$ nucleotide identity to IFNA and only about $45 \%$ nucleotide identity to IFNB. They are clearly defined on phylogenetic trees based on their ORF as a distinct clade that is most closely related to IFNA; however, phylogenetic analyses are conflicting with regard to when IFNX first appeared. Phylogenetic trees calculated on the assumption that all nucleotide sites within the coding sequence change at the same rate indicate IFNA separated from IFNX prior to or corresponding with the radiation of eutherian mammals, i.e. more than 150 MYA [35] (Fig. $5 \mathrm{~A})$. Conversely, phylogenetic trees that take into account substitution rate variation from site to site indicate that bovine IFNX and bovine IFNA emerged from a common ancestor after the radiation of the major mammalian orders (Fig. 5B). In other words, this model recognizes that certain amino acids and, by corollary certain nucleotides, are more highly conserved than others across subfamilies. This second model most closely matches the amino acid differences among IFN subfamilies, since amino acids critical to preserving the tertiary structures of IFNB, IFNA, IFNT, and IFNL are much more highly conserved than ones in less critical regions of the proteins $[55,70]$. Preliminary examination of IFNX indicate some of the more highly conserved amino acids for other Type I IFN, such as cysteine residues 1 and 99 (discussed below), are also conserved in IFNX. In addition IFNX and the IFNX pseudogene are absent in human, mouse, porcine, feline, and canine genomic databases, yet some remnants of their presence might be expected if this family emerged early in the evolution of mammals and prior to the main radiation events.

Two of the three potential IFNX genes contain the four conserved cysteine residues required for the disulfide bonds (1-99; 29-139) encountered in IFNA and those Type I subfamilies that emerged from IFNA, namely IFNW, IFND, and IFNT. The third potential gene, GLEAN 24316 [Bovine Genome Database temporary ID:2755], contains an early termination codon at codon 125 , which eliminates the second disulfide bond (29-139) (Fig. 6), raising the possibility that it is a second pseudogene. The "trace-WGS" database was visually examined to verify the presence of this early stop codon. Five BACs, all of which were in the minus orientation, contained sequences that exactly matched the complementary sequence to Glean 24316 , i.e. all contained the early termination codon.

Previous studies of IFNA have identified three regions that are strongly associated with IFN-receptor interaction and are termed interferon receptor recognition peptides (IRRP)1-3 [70-72]. IRRP1 (27-35) and IRRP2 (78-105) control the initial binding of IFN to the Type I receptor and are highly conserved among IFNA. IRRP3 (123-140) modulates the downstream signaling pathways, so that amino acid changes in this region can explain some differ- 
Table 6: Selection in IFNB.

\begin{tabular}{|c|c|c|c|c|c|c|}
\hline & 24309 & 24311 & 24313 & 24315 & 24317 & 24318 \\
\hline \multicolumn{7}{|l|}{ IFNB (24309) } \\
\hline IFNB (243II) & 0.486 & & & & & \\
\hline IFNB $(243$ I3) & 1.000 & 1.000 & & & & \\
\hline IFNB (243 I5) & $\underline{0.032}$ & 0.339 & 0.342 & & & \\
\hline IFNB (243 I7) & $\overline{0.303}$ & 1.000 & 1.000 & $\underline{0.036}$ & & \\
\hline IFNB (243 I 8) & 0.150 & 1.000 & 1.000 & 0.008 & $\underline{0.050}$ & \\
\hline
\end{tabular}

Pairwise comparisons between IFNB through the codon based Z-test are shown in the above table with the statistically significant comparisons underlined.

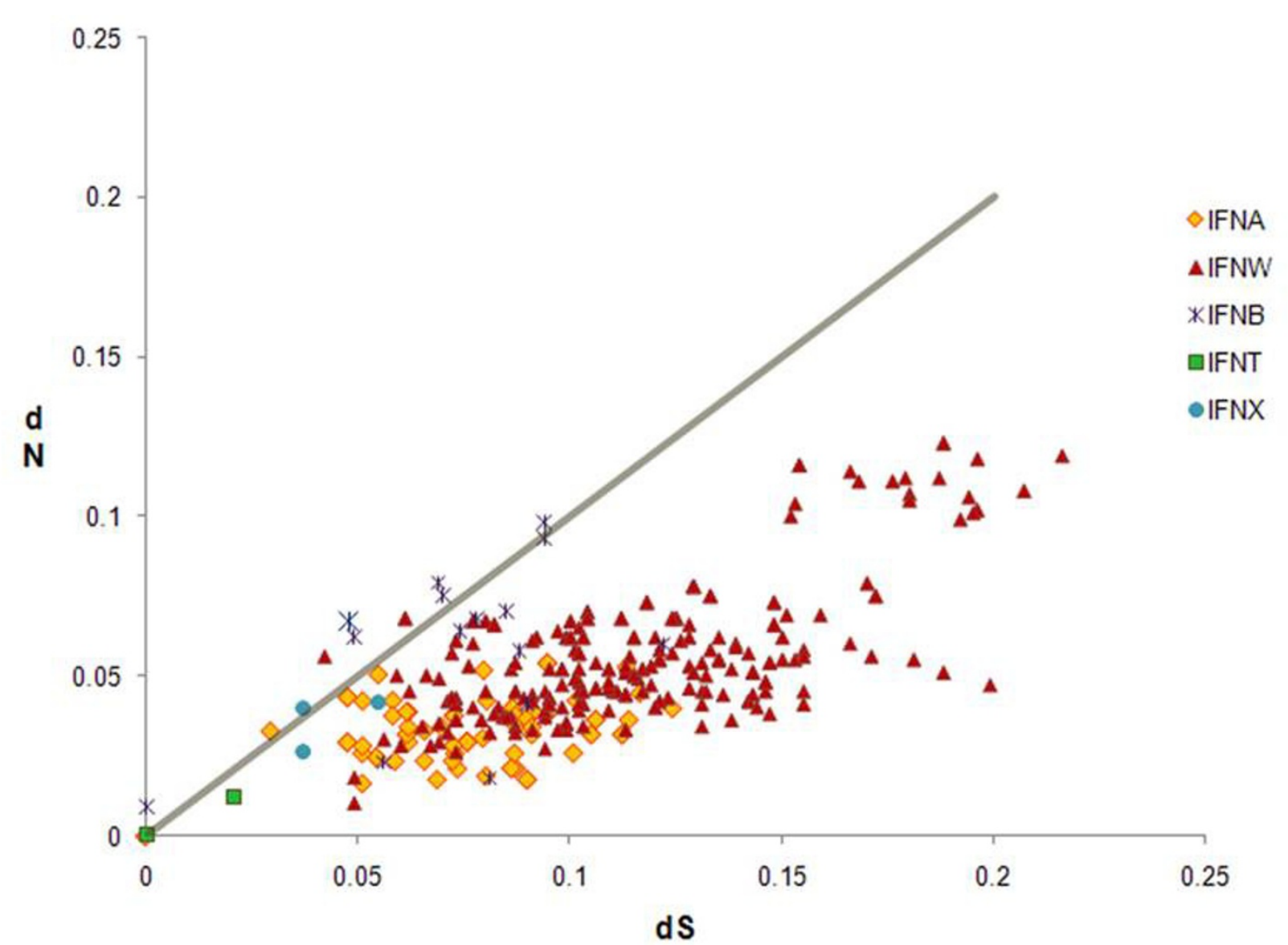

\section{Figure 4}

Selective Pressure on the coding regions of bovine Type I IFN. Pairwise analysis of the coding region between every gene pair in a Type I IFN subfamily was used to calculate dS and dN, and the two values were plotted against one another. No comparisons were made between genes belonging to different subfamilies, i.e. an IFNB gene and an IFNA gene. In addition, all probable pseudogenes, which contained early stop codons or frameshift mutations, were not included in the analysis. The grey diagonal line in the graph represent neutral selection rate where $\mathrm{dS}=\mathrm{dN}$. Gene pairs undergoing positive selection would appear above the diagonal and gene pairs undergoing purifying selection would appear below the diagonal. The graph clearly shows that no positive selection is occurring within the coding regions of any Type I IFN family. Furthermore, IFNW and IFNA subfamilies appear to be undergoing purifying selection. 

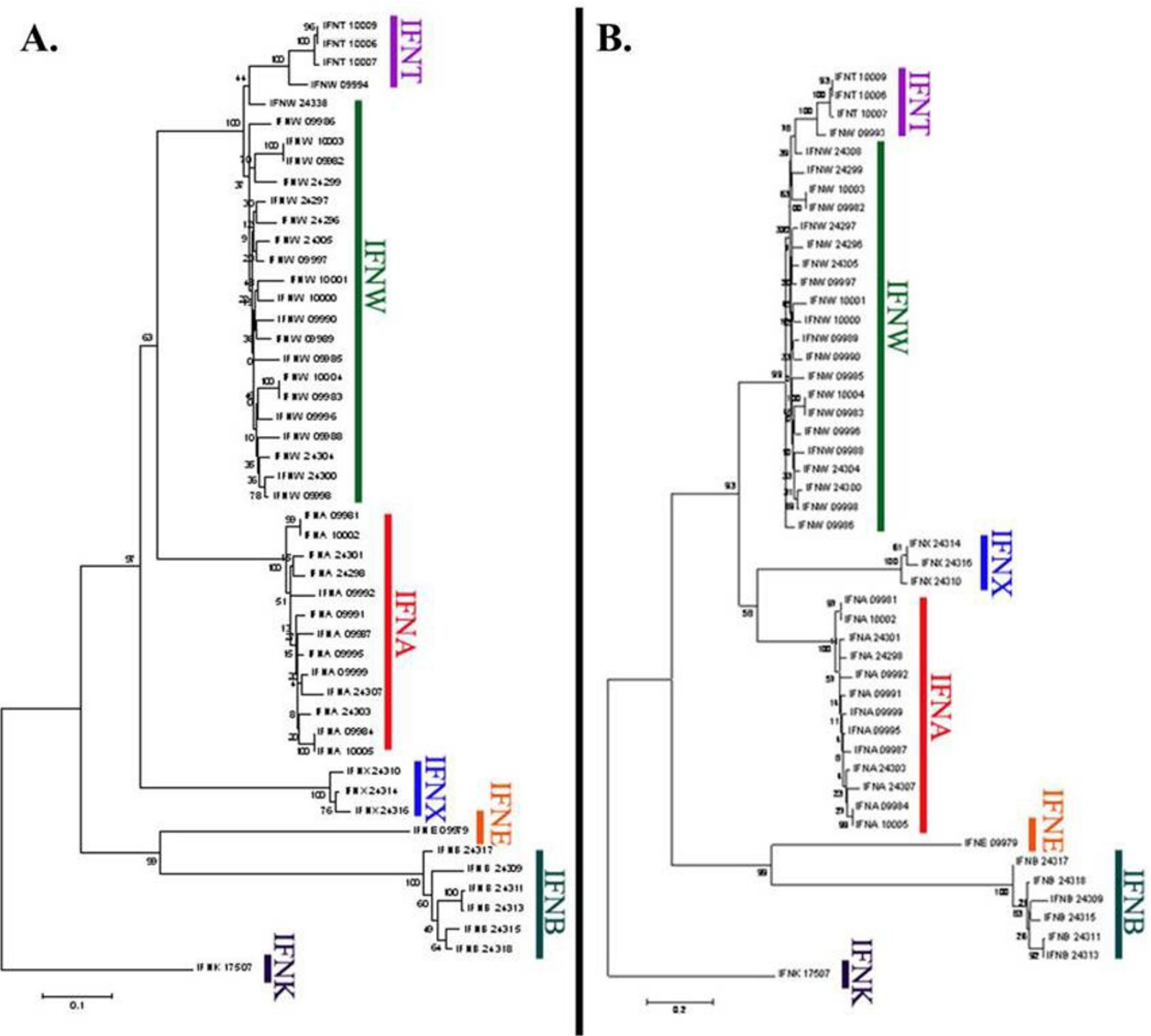

\section{Figure 5}

The Bovine Type I IFN Phylogenetic Tree. (A) The evolutionary history of bovine Type I IFN was inferred by using the Neighbor-Joining ( $\mathrm{NJ}$ ) method with bootstrap test (1000 replicates). The tree was rooted to IFNK and calculations were based on uniform rates of change for all sites. IFNX emerged prior to IFNA in this analysis. (B) The tree illustrates the evolutionary history of bovine IFN based on different rates of change between sites (gamma = I). The tree was again based on the NJ method with bootstrap test (I000 replicates) and rooted to IFNK. IFNX and IFNA branched from a common ancestor in this analysis.

ences in biological activity among different IFNA. The protein products of the three IFNX do not possess identical IRRP1 and IRRP2 motifs as IFNA, but these two regions are highly conserved within the subfamily, emphasizing, first, the possible importance of this motif and second that the IFNX family is unique and distinct from IFNA. IRRP3 was absent in GLEAN 24316 again suggesting that it may be a pseudogene. The two remaining IFNX members differed in their IRRP3 sequences, a not unexpected finding as changes in this region may provide subtle differences in biologic activity between the two family members. None of the IFNX genes contain the Nglycosylation sequence (N-X-S/T) common in other Type I IFNs that could alter IFN-receptor interaction.

No evidence for IFNX expression could be found in any EST databases, although, genes with high identity to IFNX exist in the equine genomic database. The conservation of 

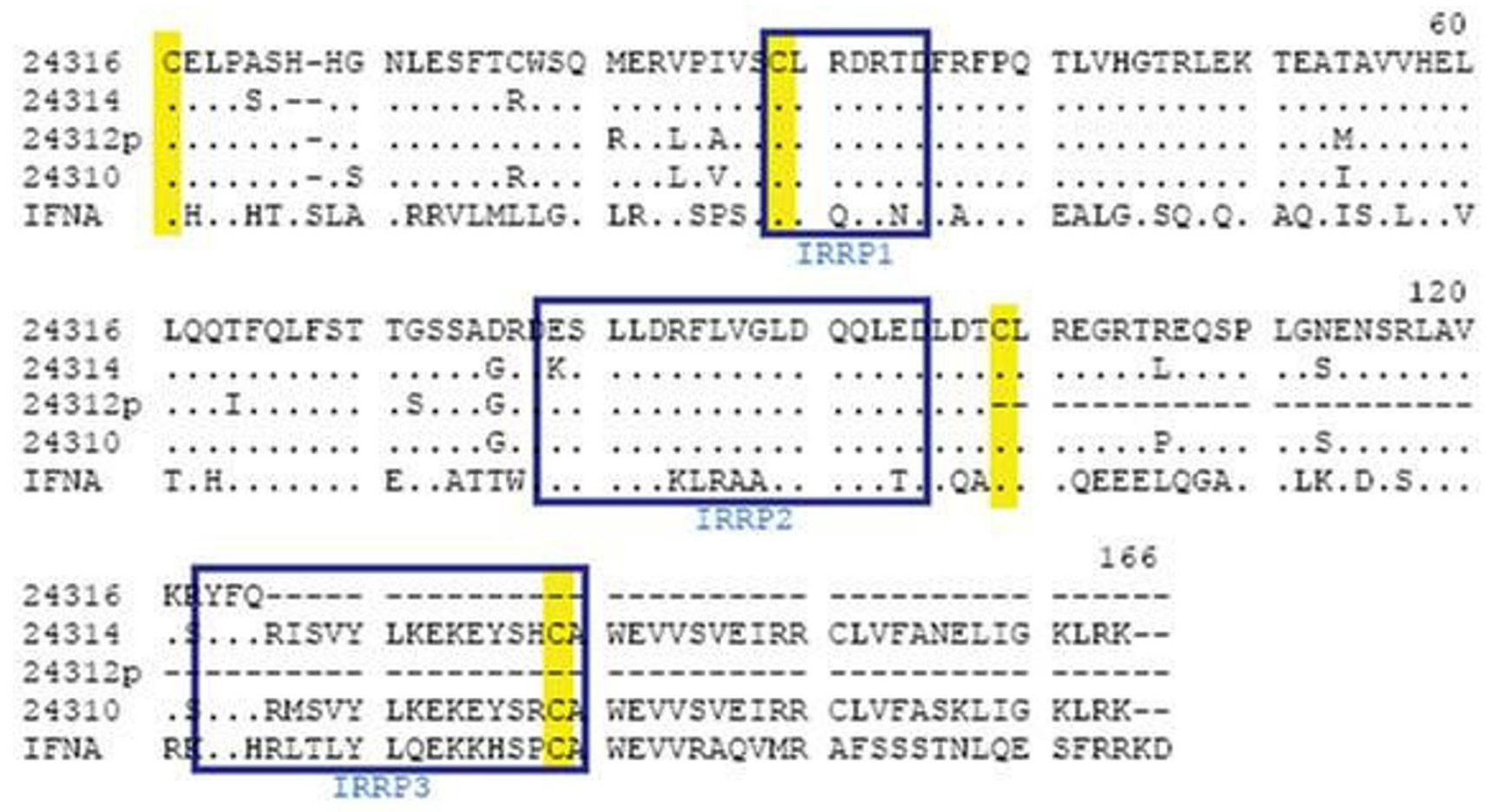

\section{Figure 6}

IFNX alignment. The coding region, minus the predicted signal peptide, for the three predicted IFNX genes are denoted by their GLEAN numbers (243 I 4 [Bovine Genome Database temporary ID:2633], 243 I0 [Bovine Genome Database temporary ID:2546], and 24316 [Bovine Genome Database temporary ID:2733]) and are aligned with bovine IFNA [Genbank:DQ396807]. The mature coding sequence of the three potentially expressed IFNX genes differs as follows 164 amino acids (aa) for Glean 24310, 163 aa for Glean 243 I4, and I 24 aa for 24316. Conserved cysteine residues form disulfide bonds in IFNA between positions $\mathrm{I} \rightarrow 99$ and $29 \rightarrow \mathrm{I} 39$ and are shown in yellow. Glean 24316 has an early stop codon that eliminates the second disulfide bond, but both Glean 24314 and Glean 24310 encode these conserved residues. Regions strongly associated with receptor binding in IFNA, or interferon receptor recognition peptides (IRRP), are boxed in blue.

this gene family in species that diverged at least 80 million years ago suggests that the family may have an important function in ungulates. However, the apparent absence of IFNX genes in pigs, also an ungulate, is puzzling. Possibly, IFNX has a specific function in herbivores that is not required in omnivores, most likely in immune defense against particular viruses or other pathogenic organisms affecting such species.

The identification of a novel Type I IFN gene, the IFNX, is an unexpected and possibly important finding. The proteins encoded by this family of genes differ sufficiently in primary sequence from related Type I IFN to justify a separate designation from the related IFNA and IFNB. The presence of a distinct cluster of IFNX within the Type I IFN locus, the phylogenetic position of IFNX as a separate clade within the IFN tree, and the conservation of critical amino acid residues, are totally consistent with classifying the IFNX as a distinct Type I IFN subfamily. Whether IFNX are responsive to a viral challenge and able to interact with the Type I IFN receptor and elicit a typical Type I response in their target cells has yet to be verified. Substantial work will be necessary to characterize this subfamily fully, but its place as a separate clade within the Type I IFN would appear to be assured.

\section{Conclusion}

The Type I IFN locus has undergone substantial transformation in ruminants compared to humans and mice. The conserved locus structure has been transformed, subfamilies have expanded, and two subfamilies not present in either humans or mice exist. The division of the locus into two sub-loci may have provided an opportunity for genes to duplicate and contribute to an expanded function of the Type I IFN. The divergence of the successful pecoran ruminant sub-order and its geographic spread might have required improved protection against unique ruminant pathogens. The IFNX sub-family and the greatly expanded ruminant specific IFNW are likely candidates for providing such protection. Radically new functions for 
Type I IFN might also have been gained, such as the one exemplified by the IFNT, whose appearance coincided with, and possibly permitted, the acquisition of the unique, synepitheliochorial placentation that characterizes the Ruminantia sub-order and requires powerful conceptus signaling before the trophoblast has even attached to the uterine wall [24]. The ancient Type III IFN (IFNL/ IL28-29) may have become a casualty of the expansion and broadened the role of the Type I locus, as only an inactive IFNL remains in the bovine genome. It is tempting to speculate that the function of IFNL has been replaced as the component genes of the Type I IFN locus expanded.

The authors concede that the bovine genome assembly is a work in progress and that the predicted arrangement of individual IFN genes may have to be modified as data are reanalyzed. In addition, it is clear that an individual animal possesses unique genomic peculiarities, including inversions, duplications, and presence and absence of specific genes and that the IFN locus of a single Hereford cow may not be replicated precisely in other breeds. Nevertheless, with the exception of the size of the "gap" between the two sub-loci, the organization and sequence of the bovine Type I IFN have remained relatively constant through the most recent assemblies. The unique features of the locus, which include the presence of the gap itself, the arrangements of IFNW/IFNA clusters, the dramatic expansion of the IFNW, the presence of the IFNX, and the separation of IFNT from the IFNW/IFNA clusters are consistent observations and unlikely to undergo drastic reevaluation in future versions of the assembly.

\section{Authors' contributions}

AMW designed the study and performed all bioinformatics. RMR proposed and supervised the study. Both authors wrote, read and approved the manuscript.

\section{Acknowledgements}

We would like to thank the Bovine Genome Sequencing and Annotation Consortium for coordinating with this project and providing Apollo training. We would also like to thank Drs. Jonathan Green and Bhanu Telugu for advice on the data analysis (bioinformatics). Funding for this work was provided by $\mathrm{NIH}$ grant HD21896.

\section{References}

I. Goodbourn S, Didcock L, Randall RE: Interferons: cell signalling, immune modulation, antiviral response and virus countermeasures. J Gen Virol 2000, 8 I:234I-2364.

2. lannello A, Debbeche O, Martin E, Attalah LH, Samarani S, Ahmad A: Viral strategies for evading antiviral cellular immune responses of the host. J Leukoc Biol 2006, 79:16-35.

3. Randall RE, Goodbourn S: Interferons and viruses: an interplay between induction, signalling, antiviral responses and virus countermeasures. J Gen Virol 2008, 89: I-47.

4. Stark GR, Kerr IM, Williams BR, Silverman RH, Schreiber RD: How cells respond to interferons. Annu Rev Biochem 1998, 67:227-264.

5. Krause CD, Pestka S: Evolution of the Class 2 cytokines and receptors, and discovery of new friends and relatives. Pharmacol Ther 2005, 106:299-346.
6. Levraud JP, Boudinot P, Colin I, Benmansour A, Peyrieras N, Herbomel $P$, Lutfalla G: Identification of the zebrafish IFN receptor: implications for the origin of the vertebrate IFN system. J Immunol 2007, 178:4385-4394.

7. Lutfalla G, Roest Crollius H, Stange-Thomann N, Jaillon O, Mogensen $\mathrm{K}$, Monneron D: Comparative genomic analysis reveals independent expansion of a lineage-specific gene family in vertebrates: the class II cytokine receptors and their ligands in mammals and fish. BMC Genomics 2003, 4:29.

8. Hedges SB: The origin and evolution of model organisms. Nat Rev Genet 2002, 3:838-849.

9. Uze G, Monneron D: IL-28 and IL-29: newcomers to the interferon family. Biochimie 2007, 89:729-734.

10. Sheppard P, Kindsvogel W, Xu W, Henderson K, Schlutsmeyer S, Whitmore TE, Kuestner R, Garrigues U, Birks C, Roraback J, et al:: IL-28, IL-29 and their class II cytokine receptor IL-28R. Nat Immunol 2003, 4:63-68.

II. Ank N, Iversen MB, Bartholdy C, Staeheli P, Hartmann R, Jensen UB, Dagnaes-Hansen F, Thomsen AR, Chen Z, Haugen $H$, et al:: An important role for type III interferon (IFN-lambda/IL-28) in TLR-induced antiviral activity. J Immunol 2008, 180:2474-2485.

12. Kotenko SV, Gallagher G, Baurin VV, Lewis-Antes A, Shen M, Shah NK, Langer JA, Sheikh F, Dickensheets H, Donnelly RP: IFN-lambdas mediate antiviral protection through a distinct class II cytokine receptor complex. Nat Immunol 2003, 4:69-77.

13. Rubinstein M, Rubinstein S, Familletti PC, Miller RS, Waldman AA, Pestka S: Human leukocyte interferon: production, purification to homogeneity, and initial characterization. Proc Natl Acad Sci USA 1979, 76:640-644.

14. Stein S, Kenny C, Friesen HJ, Shively J, Del Valle U, Pestka S: NH2terminal amino acid sequence of human fibroblast interferon. Proc Natl Acad Sci USA 1980, 77:5716-5719.

15. Pestka $S:$ The interferons: $\mathbf{5 0}$ years after their discovery, there is much more to learn. J Biol Chem 2007, 282:20047-2005I.

16. Pestka $S$, Krause $C D$, Walter MR: Interferons, interferon-like cytokines, and their receptors. Immunol Rev 2004, 202:8-32.

17. Barnes B, Lubyova B, Pitha PM: On the role of IRF in host defense. J Interferon Cytokine Res 2002, 22:59-7I.

18. Paltrinieri S, Crippa A, Comerio T, Angioletti A, Roccabianca P: Evaluation of inflammation and immunity in cats with spontaneous parvovirus infection: consequences of recombinant feline interferon-omega administration. Vet Immunol Immunopathol 2007, II 8:68-74.

19. de Mari K, Maynard L, Eun HM, Lebreux B: Treatment of canine parvoviral enteritis with interferon-omega in a placebo-controlled field trial. Vet Rec 2003, I 52:105-108.

20. Oritani K, Medina KL, Tomiyama Y, Ishikawa J, Okajima Y, Ogawa M, Yokota T, Aoyama K, Takahashi I, Kincade PW, et al: Limitin: An interferon-like cytokine that preferentially influences $\mathrm{B}$-lymphocyte precursors. Nat Med 2000, 6:659-666.

21. Buontempo PJ, Jubin RG, Buontempo CA, Wagner NE, Reyes GR, Baroudy BM: Antiviral activity of transiently expressed IFNkappa is cell-associated. J Interferon Cytokine Res 2006, 26:40-52.

22. LaFleur DW, Nardelli B, Tsareva T, Mather D, Feng P, Semenuk M, Taylor K, Buergin M, Chinchilla D, Roshke V, et al.: Interferonkappa, a novel type I interferon expressed in human keratinocytes. J Biol Chem 200I, 276:39765-3977I.

23. Matsumiya T, Prescott SM, Stafforini DM: IFN-epsilon mediates TNF-alpha-induced STATI phosphorylation and induction of retinoic acid-inducible gene-I in human cervical cancer cells. J Immunol 2007, I 79:4542-4549.

24. Roberts RM, Chen Y, Ezashi T, Walker AM: Interferons and the maternal-conceptus dialog in mammals. Semin Cell Dev Biol 2008, 19:170-177.

25. Lefevre F, Guillomot M, D'Andrea S, Battegay S, La Bonnardiere C: Interferon-delta: the first member of a novel type $I$ interferon family. Biochimie 1998, 80:779-788.

26. Trent JM, Olson S, Lawn RM: Chromosomal localization of human leukocyte, fibroblast, and immune interferon genes by means of in situ hybridization. Proc Natl Acad Sci USA 1982, 79:7809-78I3.

27. Hardy MP, Owczarek CM, Jermiin LS, Ejdeback M, Hertzog PJ: Characterization of the type I interferon locus and identification of novel genes. Genomics 2004, 84:33I-345.

28. Diaz MO, Pomykala HM, Bohlander SK, Maltepe E, Malik K, Brownstein B, Olopade OI: Structure of the human type-I interferon 
gene cluster determined from a YAC clone contig. Genomics 1994, 22:540-552.

29. Takahashi I, Kosaka H, Oritani K, Heath WR, Ishikawa J, Okajima $Y$, Ogawa M, Kawamoto S, Yamada M, Azukizawa H, et al.: A new IFNlike cytokine, limitin, modulates the immune response without influencing thymocyte development. J Immunol 200I, 167:3156-3163.

30. Hughes AL: The evolution of the type I interferon gene family in mammals. I Mol Evol 1995, 41:539-548.

31. Yang LM, Xue QH, Sun L, Zhu YP, Liu WJ: Cloning and characterization of a novel feline IFN-omega. J Interferon Cytokine Res 2007, 27: I19-127.

32. Roberts RM, Ezashi T, Rosenfeld CS, Ealy AD, Kubisch HM: Evolution of the interferon tau genes and their promoters, and maternal-trophoblast interactions in control of their expression. Reprod Suppl 2003, 61:239-25I.

33. Leaman DW, Roberts RM: Genes for the trophoblast interferons in sheep, goat, and musk ox and distribution of related genes among mammals. J Interferon Res 1992, I 2: I-II.

34. Capon DJ, Shepard HM, Goeddel DV: Two distinct families of human and bovine interferon-alpha genes are coordinately expressed and encode functional polypeptides. Mol Cell Biol 1985, 5:768-779.

35. Roberts RM, Liu L, Guo Q, Leaman D, Bixby J: The evolution of the type I interferons. J Interferon Cytokine Res 1998, I8:805-816.

36. lannuzzi L, Gallagher DS, Ryan AM, Di Meo GP, Womack JE: Chromosomal localization of omega and trophoblast interferon genes in cattle and river buffalo by sequential R-banding and fluorescent in situ hybridization. Cytogenet Cell Genet 1993, 62:224-227.

37. Ryan AM, Gallagher DS, Womack JE: Somatic cell mapping of omega and trophoblast interferon genes to bovine syntenic group $\mathrm{U} / 8$ and in situ localization to chromosome 8. Cytogenet Cell Genet 1993, 63:6-10.

38. Roberts RM, Leaman DW, Cross JC: Role of interferons in maternal recognition of pregnancy in ruminants. Proc Soc Exp Biol Med 1992, 200:7-18.

39. NCBI/BLAST Cow Sequences [http://www.ncbi.nlm.nih.gov/ genome/seq/BlastGen/BlastGen.cgi!taxid=99/3]

40. NCBI/Mapviewer Home [http://www.ncbi.nlm.nih.gov/mapview/]

4I. Ye J, McGinnis S, Madden TL: BLAST: improvements for better sequence analysis. Nucleic Acids Res 2006, 34:W6-9.

42. NCBI/BLAST Home [http://www.ncbi.nlm.nih.gov/blast/ Blast.cgi?CMD=Web\&PAGE TYPE=BlastHome]

43. McEntyre J, (ed): The NCBI Handbook [Internet]. Bethesda, MD: The National Library of Medicine (US), National Center for Biotechnology Information; 2002.

44. Elsik CG, Mackey AJ, Reese JT, Milshina NV, Roos DS, Weinstock GM: Creating a honey bee consensus gene set. Genome Biol 2007, 8:RI3

45. Apollo Genome Annotation Curation Tool [http://apollo.ber keleybop.org/current/index.html]

46. Lewis SE, Searle SM, Harris N, Gibson M, Lyer V, Richter J, Wiel C, Bayraktaroglir L, Birney E, Crosby MA, et al.: Apollo: a sequence annotation editor. Genome Biol 2002, 3:RESEARCH0082.

47. Biological sequence alignment editor for Win95/98/NT/2K/ XP [http://www.mbio.ncsu.edu/BioEdit/BioEdit.html]

48. Thompson JD, Higgins DG, Gibson TJ: CLUSTAL W: improving the sensitivity of progressive multiple sequence alignment through sequence weighting, position-specific gap penalties and weight matrix choice. Nucleic Acids Res 1994, 22:4673-4680.

49. Molecular Evolutionary Genetics Analysis [http:// www.megasoftware.net]

50. Tamura K, Dudley J, Nei M, Kumar S: MEGA4: Molecular Evolutionary Genetics Analysis (MEGA) software version 4.0. Mol Biol Evol 2007, 24: I596-1599.

51. Tamura K, Nei M, Kumar S: Prospects for inferring very large phylogenies by using the neighbor-joining method. Proc Natl Acad Sci USA 2004, I01: I 1030-I I035.

52. RepeatMasker [http://www.repeatmasker.org/]

53. Jurka J, Kapitonov VV, Pavlicek A, Klonowski P, Kohany O, Walichiewicz J: Repbase Update, a database of eukaryotic repetitive elements. Cytogenet Genome Res 2005, I I 0:462-467.

54. Washington University in St. Louis WU-Blast [http:// blast.wustl.edu/]
55. Oritani K, Kincade PW, Zhang C, Tomiyama Y, Matsuzawa Y: Type $I$ interferons and limitin: a comparison of structures, receptors, and functions. Cytokine Growth Factor Rev 200 I, I 2:337-348.

56. Lefevre F, Boulay V: A novel and atypical type one interferon gene expressed by trophoblast during early pregnancy. J Biol Chem 1993, 268:19760-19768.

57. Ealy AD, Larson SF, Liu L, Alexenko AP, Winkelman GL, Kubisch HM, Bixby JA, Roberts RM: Polymorphic forms of expressed bovine interferon-tau genes: relative transcript abundance during early placental development, promoter sequences of genes and biological activity of protein products. Endocrinology 200 I, | 42:2906-29|5

58. Yang S, Arguello JR, Li X, Ding Y, Zhou Q, Chen Y, Zhang Y, Zhao R, Brunet $F$, Peng $L$, et al.: Repetitive element-mediated recombination as a mechanism for new gene origination in Drosophila. PLoS Genet 2008, 4:e3.

59. Fiston-Lavier AS, Anxolabehere D, Quesneville $\mathrm{H}$ : A model of segmental duplication formation in Drosophila melanogaster. Genome Res 2007, 17:1458-1470.

60. Apostolou E, Thanos D: Virus Infection Induces NF-kappaBdependent interchromosomal associations mediating monoallelic IFN-beta gene expression. Cell 2008, 134:85-96.

6I. Woelk CH, Frost SD, Richman DD, Higley PE, Kosakovsky Pond SL: Evolution of the interferon alpha gene family in eutherian mammals. Gene 2007, 397:38-50.

62. Miyata $\mathrm{T}$, Hayashida $\mathrm{H}$, Kikuno $\mathrm{R}$, Toh $\mathrm{H}$, Kawade $\mathrm{Y}$ : Evolution of interferon genes. Interferon 1985, 6: I-30.

63. Chen JM, Cooper DN, Chuzhanova N, Ferec C, Patrinos GP: Gene conversion: mechanisms, evolution and human disease. Nat Rev Genet 2007, 8:762-775.

64. Blanco P, Shlumukova M, Sargent CA, Jobling MA, Affara N, Hurles ME: Divergent outcomes of intrachromosomal recombination on the human $\mathbf{Y}$ chromosome: male infertility and recurrent polymorphism. J Med Genet 2000, 37:752-758.

65. Hurles M: Gene duplication: the genomic trade in spare parts. PLoS Biol 2004, 2:E206.

66. Hughes AL: The evolution of functionally novel proteins after gene duplication. Proc Biol Sci 1994, 256: I 19-124.

67. Cheng G, Chen W, Li Z, Yan W, Zhao X, Xie J, Liu M, Zhang H, Zhong Y, Zheng Z: Characterization of the porcine alpha interferon multigene family. Gene 2006, 382:28-38.

68. Walsh JB: How often do duplicated genes evolve new functions? Genetics 1995, 139:421-428.

69. Ohno S: Evolution by gene duplication. Ist edition. New York, New York: Springer-Verlag; 1970.

70. Kumaran J, Wei L, Kotra LP, Fish EN: A structural basis for interferon-alpha-receptor interactions. FASEB / 2007, 21:3288-3296.

71. Piehler J, Schreiber G: Biophysical analysis of the interaction of human ifnar2 expressed in E. coli with IFNalpha2. J Mol Biol 1999, 289:57-67.

72. Chill JH, Nivasch R, Levy R, Albeck S, Schreiber G, Anglister J: The human interferon receptor: NMR-based modeling, mapping of the IFN-alpha 2 binding site, and observed ligand-induced tightening. Biochemistry 2002, 41:3575-3585.

Publish with Biomed Central and every scientist can read your work free of charge

"BioMed Central will be the most significant development for disseminating the results of biomedical research in our lifetime. "

Sir Paul Nurse, Cancer Research UK

Your research papers will be:

- available free of charge to the entire biomedical community

- peer reviewed and published immediately upon acceptance

- cited in PubMed and archived on PubMed Central

- yours - you keep the copyright 